concluyó que aunque $\mathrm{M}$ redujo peso, dosis de insulina y LDL colesterol, no mejoró el control glucémico en pacientes adultos con DM1 de larga evolución, aunque podría jugar un rol pequeño en la prevención de eventos cardiovasculares. Los tres IDPP-4 más conocidos se utilizaron por $<1 \%$ en el T1DE, sin cambios significativos en control metabólico y muy discreto impacto en el peso. Los AGLP-1 empleados por el $6 \%$ de los DM1 en elT1DE, si bien demostraron tanto con dosis de 1,2 y 1,8 mg/día disminución significativa de dosis de insulina y de peso, no redujeron los eventos hipoglucémicos, no mejoraron la secreción de hormonas contrinsulares ni la respuesta clínica a hipoglucemias, generando por otra parte un porcentaje importante de eventos gastrointestinales.

Finalmente los ISGLT-1 e ISGLT duales se evaluaron en pacientes con DM1. Por su particular mecanismo de acción (dapagliflozina, canagliflozina, empagliflozina) ofrecen una alternativa complementaria muy interesante y generan disminución de peso, de hipoglucemias de dosis total de insulina, de variabilidad glucémica y de A1c (empagliflozina, en ocho semanas, generó versus placebo una reducción de $-0,4 \%$ en A1c, $36 \mathrm{mg} / \mathrm{dl}$ en el promedio de glucemias del monitoreo, 2,7 Kg y $33 \mathrm{ml} / \mathrm{m}$ del filtrado glomerular en pacientes con $>135 \mathrm{ml} / \mathrm{m}$ en el inicio del estudio). A tal punto convincentes que ya se aprobó en la European Medicines Agency (EMA) el uso de dapagliflozina en DM1. Es importante advertir los efectos colaterales como infecciones urinarias y genitales, sobre todo en mujeres. El mayor riesgo para el uso de este tipo de medicación radica en la incidencia de cetoacidosis diabética aumentada. Es necesario indicar a los pacientes que no deben reducir la dosis de insulina sin consultar, e identificar aquellos con mayor riesgo de padecer esta complicación. La sotagliflozina es un ISGLT-1 y 2; tiene un efecto intestina adicional lo cual potencia su acción sobre todo en glucemias postprandiales y menor variabilidad. Sin embargo está en duda si se empleará dado que el estudio con sotagliflozina se interrumpió en DM1 por la mayor incidencia de cetoacidosis.

Concluimos que si bien la insulina es la medicación necesaria y adecuada para pacientes con DM1, la presencia de hipoglucemias, aumento de peso y mayor prevalencia de SM en estos pacientes indica la necesidad de complementar dicho tratamiento con otros fármacos. Hasta el momento los únicos autorizados son pramlintida y dapagliflozina. El seguimiento de varios estudios en marcha probablemente aclarará el rol principalmente de los AGLP-1 e ISGLT-2 en pacientes con DM1.

\title{
BIBLIOGRAFÍA
}

- Bode BW, et. al. Adjuntive therapy for type 1 diabetes. Endocr Pract 2016; 22 (2).

- Miller KM, Foster NC, Beck RW, et al. Current state of type 1 diabetes treatment in the U.S.: updated data from the T1D Exchange clinic registry. Diabetes Care 2015; 38:971-978.

- National Institutes of Health. ClinicalTrials.gov. Disponible en: https://clinicaltrials.gov.

- Standl E, et al. Metformin in type 1 diabetes. The Lancet Diabetes \& Endocrinology 2017; Vol 5, Issue 8: 567-569.

\section{Tratamiento de la obesidad en diabetes mellitus tipo 1}

\author{
Dra. Carla Musso
}

El aumento de la prevalencia de obesidad en diabetes mellitus tipo 1 (DM1) es alarmante y se asocia a insulinorresistencia semejante a lo observado en DM2, lo cual generó considerarla "diabetes doble". Actualmente el $50 \%$ de los pacientes con DM1 tiene sobrepeso u obesidad. También la asociación con obesidad ha generado el desarrollo de dislipemia y complicaciones macrovasculares. Las comorbilidades asociadas a la obesidad reducen los beneficios del buen control metabólico, razón por la cual es importante el objetivo del normopeso. El estado proinflamatorio crónico y sostenido de la obesidad se asocia con enfermedad cardiovascular.

Las estrategias a implementar incluyen el plan de alimentación reducido en calorías, bajo en hidratos de carbono e índice glucémico, y elevado en fibras y proteínas magras. La actividad física aeróbica y anaeróbica debe indicarse para mantener la masa muscular. Es importante realizar ajustes de dosis de insulina durante el descenso de peso.

Debe considerarse medicación específica para el manejo de la obesidad y en caso de no lograr los objetivos es adecuado sugerir la cirugía metabólica. La medicación utilizada con resultados variables ha sido metformina, los agonistas del receptor de GLP-1, pramlintida e inhibidores de SGLT-2, si bien la implementación de las mismas aún no ha sido aprobada en DM1 por la Food and Drug Administration (FDA). 
Medicaciones antiobesidad como topiramato/fentermina, lorcaserina, naltrexona/bupropión, orlistat y fentermina son efectivas pero no existen estudios realizados específicamente en pacientes con DM1.

En cuanto a la cirugía metabólica ambas técnicas, by pass en Y de Roux y manga gástrica, se implementaron en pacientes con DM1 con resultados discutibles.

Es necesario generar guías de estrategias para prevenir y controlar la ganancia de peso en pacientes con DM1 que requieran tratamiento insulínico intensificado.

\section{BIBLIOGRAFÍA}

- Corbin KD, Driscoll KA, Pratley RE, Smith SR, Maahs DM, Mayer-Davis EJ; Advancing Care forType 1 Diabetes and Obesity Network (ACT1ON). Obesity in type 1 diabetes: pathophysiology, clinical impact, and mechanisms. Endocrine Reviews 2018; 39: 629-663.

- Polskya S, Ellisb SL. Obesity, insulin resistance, and type 1 diabetes mellitus. Curr Opin Endocrinol Diabetes Obes 2015; 22:277-282.

- Mottalib A, Kasetty M, Mar JY, ElseaidyT, Ashrafzadeh S, Hamdy O. Weight management in patients with type 1 diabetes and obesity. Curr Diab Rep 2017 Aug 23; 17(10):92. Doi: 10.1007/s11892-017-0918-8.

\section{Enfermedad celíaca y diabetes mellitus tipo 1}

Dra. Mabel Ferraro

La joven nieta de Juana (21 años) es portadora de diabetes mellitus tipo 1 (DM1), obesidad y enfermedad celíaca $(E C)$. La EC se origina por la reacción inmune mediada por células $T$ al gluten de la dieta y se caracteriza por la inflamación del intestino delgado, atrofia vellosa, hiperplasia de la cripta y malabsorción. La prevalencia de esta asociación es del $1 \%$ a nivel mundial, muy variable en los diferentes países y asciende del 4 al 10\% si se asocia con DM11. La base genética de la misma es un HLA característico DQ2/DQ8 necesario pero no excluyente para su aparición. Se conocen otros genes no HLA de susceptibilidad como CTLA-4, PTPN22, VDR, IL-2Ra y TNF- $\alpha$ que pudiera presentar esta paciente ${ }^{2}$.

En los últimos años se ha revalorizado el papel de la microbiota como protectora o favorecedora de su aparición. Sería interesante recabar si la joven nació por vía vaginal, recibió lactancia materna y el momento de incorporación del gluten. La presencia de bifidobacterias y lactobacilos podría haber disminuido el riesgo ${ }^{3}$. El diagnóstico debió realizarse por pesquisa sistemática indispensable en los portadores de DM1. Según la International Society for Pediatric and Adolescent Diabetes (ISPAD) la misma debe realizarse al debut, anualmente en los primeros años y luego cada cinco años o cuando exista sospecha4.

La EC se asocia a otras inmunopatías como tiroiditis, hipoparatiroidismo ideopático, enfermedad de Addison y síndrome poliglandular autoinmune. Para la pesquisa de EC se solicitan Ac específicos como antitransglutaminasa, péptidos desaminados de gliadina o antiendomisio según experiencia y disponibilidad, y dosaje de IgA para descartar deficiencia y falsos resultados negativos. La confirmación diagnóstica se realiza por biopsia duodenal. Algunos autores sostienen que puede prescindirse de la misma si el título de anticuerpos es muy elevado (más de 10 veces el valor base) ${ }^{5}$.

En nuestra paciente es importante el seguimiento en cuanto a evolución y adherencia. Debe monitorearse el riesgo de aterosclerosis temprana y de complicaciones a largo plazo. Si bien no existen datos concluyentes, este grupo podría presentar un riesgo mayor favorecido por el HLA de base con una mayor tendencia a infecciones e inflamación $n^{6}$. Particularmente en esta paciente la obesidad funcionaría como una inflamación de bajo grado y un mayor riesgo adicionado de aterosclerosis.

\section{BIBLIOGRAFÍA}

1. Elfstrom P, Sundstrom J, Ludvigsson JF. Systematic review with meta-analysis: associations between coeliac disease and type 1 diabetes. Aliment PharmacolTher 2014 Nov; 40(10):1123-32.

2. Kahaly JG, Frommer L, Schuppan D. Celiac disease and endocrine autoimmunity: the genetic link. Autrev 2018. Doi:10.1016/j.autrev.2018.05.013.

3. Krishnareddy S. The microbiome in celiac disease. Gastroenterol Clin North Am 2019 Mar; 48(1):115-126. Doi: 10.1016/j. gtc.2018.09.008.

4. Pihoker C, Forsander G, Fantahun B, Virmani A, Corathers S, Benítez-Aguirre P, Fu J, Maahs DM. ISPAD Clinical Practice Consensus Guidelines 2018. The delivery of ambulatory diabetes care to children and adolescents with diabetes. Pediatr Diabetes 2018 Oct; 19 Suppl 27:84-104. Doi: 10.1111/pedi.12757. 